\title{
A sea ice concentration estimation algorithm utilizing radiometer and SAR data
}

\author{
J. Karvonen \\ Finnish Meteorological Institute (FMI), Helsinki, PB 503, 00101, Finland \\ Correspondence to: J. Karvonen (juha.karvonen@fmi.fi)
}

Received: 7 March 2014 - Published in The Cryosphere Discuss.: 28 April 2014

Revised: 24 July 2014 - Accepted: 29 July 2014 - Published: 5 September 2014

\begin{abstract}
We have studied the possibility of combining the high-resolution synthetic aperture radar (SAR) segmentation and ice concentration estimated by radiometer brightness temperatures. Here we present an algorithm for mapping a radiometer-based concentration value for each SAR segment. The concentrations are estimated by a multi-layer perceptron (MLP) neural network which has the AMSR-2 (Advanced Microwave Scanning Radiometer 2) polarization ratios and gradient ratios of four radiometer channels as its inputs. The results have been compared numerically to the gridded Finnish Meteorological Institute (FMI) ice chart concentrations and high-resolution AMSR-2 ASI (ARTIST Sea Ice) algorithm concentrations provided by the University of Hamburg and also visually to the AMSR-2 bootstrap algorithm concentrations, which are given in much coarser resolution. The differences when compared to FMI daily ice charts were on average small. When compared to ASI ice concentrations, the differences were a bit larger, but still small on average. According to our comparisons, the largest differences typically occur near the ice edge and sea-land boundary. The main advantage of combining radiometerbased ice concentration estimation and SAR segmentation seems to be a more precise estimation of the boundaries of different ice concentration zones.
\end{abstract}

\section{Introduction}

Ice concentration is defined as the ratio of the ice-covered area to the total area for a given sea region. From this definition, it directly follows that ice concentration is dependent on the resolution of the measurement. This fact also complicates direct comparison to different ice concentration products in different resolutions. Spaceborne radiometers are an important data source, capable of estimating the ice concentration at a resolution of around $10 \mathrm{~km}$ or even coarser. Also, other spaceborne instruments for estimating the ice concentration have been used. The advantage of radiometer platforms and the radiometer instruments on-board, like AMSR-2 (Advanced Microwave Scanning Radiometer 2), is that their orbit and wide swaths enable a daily coverage over most of the ice-covered sea areas. Algorithms producing ice concentration from radiometer data are, e.g., the NASA Team algorithm (Cavalieri et al., 1984), the bootstrap algorithm (Comiso, 1986, 1995) used by the National Sea Ice Data Center (NSIDC), and the Artist Sea Ice (ASI) algorithm (Kaleschke et al., 2001; Spreen et al., 2008) of the University of Bremen (UB). The ASI algorithm utilizing the full resolution of the AMSR-2 has been introduced and is operationally providing Arctic and Antarctic ice concentration at the University of Hamburg (UH) (Beitsch et al., 2013, 2014). Here we also provide a comparison of our results to these fine-resolution radiometer ice concentration estimates. Ice concentration at a finer resolution can also be retrieved from instruments capable of measuring surface temperature, but these instruments operate at (infrared) wavelengths not penetrating the cloud cover. Due to relatively long cloudy periods, which can even last several weeks, during the wintertime there long temporal gaps can occur in these measurements. One example of such algorithms is the MPA (MODIS potential open-water algorithm) (Drüe and Heinemann, 2004), developed at the University of Bonn, using the Moderate Resolution Imaging Spectroradiometer (MODIS) instrument data for ice concentration retrieval. Synthetic aperture radar (SAR) data, with high resolution, large spatial coverage and the capability to acquire data through cloud 
cover, have not yet been widely used in measuring sea ice concentration. Some studies using single-band SAR for the estimation of ice concentration have however been made, e.g. those reported in Bovith and Andersen (2005), Berg (2011), Karvonen (2012) and Karvonen et al. (2012). Automated sea ice classification schemes, which implicitly include ice concentration or an open-water class, based on single-band and dual-band SAR texture and backscattering, have also been proposed, e.g. in Clausi and Jernigan (2000), Clausi (2001), Deng and Clausi (2004, 2005), Maillard et al. (2005), Yu and Clausi (2007), Ochilov and Clausi (2012) and Leigh et al. (2014). These methods use multiple techniques, like the grey-level co-occurrence texture features (Haralick at al., 1973), Markov random fields (MRF) (Rue and Held, 2005), and Gabor filters (Pichler et al., 1996; Clausi and Jernigan, 2000) for classifying the sea ice SAR data. Dokken et al. (2000) developed a SAR ice concentration algorithm which is a combination of mean ratio (relating average SAR backscatter to typical open-water and sea ice values), a local threshold and a wavelet method (to detect edges around ice floes) for RADARSAT-1 (the first satellite of a series of Canadian earth observation satellites with a Cband SAR instrument) and ERS (European Remote Sensing satellite of the European Space Agency) SAR imagery. It has also been shown that using dual-polarized C-band SAR data $(\mathrm{HH} / \mathrm{HV})$ improves the ice concentration estimation compared to single-channel SAR (HH) (Karvonen, 2014). A method for ice classification into four ice classes and open water by combining SSM/I (Special Sensor Microwave/Imager) radiometer ice concentration and SAR data was introduced in Kaleschke and Kern (2000). Here we propose a novel method combining high-resolution SAR image segmentation and the lower-resolution radiometer ice concentration estimation to yield ice concentration estimates with improved areal boundaries defined by the SAR resolution. The algorithm is based on SAR image segmentation and on multi-layer perceptron (MLP) neural network.

\section{Study area, time and data}

We studied our novel algorithm for the Baltic Sea. The study area is defined by the upper left and lower right corner in Fig. $1\left(56.0^{\circ} \mathrm{N}, 16.0^{\circ} \mathrm{E}\right.$ and $66.0^{\circ} \mathrm{N}, 31.0^{\circ} \mathrm{E}$, respectively). The area of the Baltic Sea is $422000 \mathrm{~km}^{2}$. Baltic Sea ice cover has large annual and local variations. Annual maximum ice extent varies between 52000 and $422000 \mathrm{~km}^{2}$, the average being $218000 \mathrm{~km}^{2}$ (reached in January-March). The Bay of Bothnia and Gulf of Finland have at least partial ice cover every year. Baltic Sea ice moves and the ice concentration changes during each winter; also, some melting can occur even in midwinter because the temperature may also vary from -40 to $+10^{\circ} \mathrm{C}$ even during midwinter. Only the landfast ice areas covering a relatively narrow zone along the coasts (from a few kilometres to a few tens of

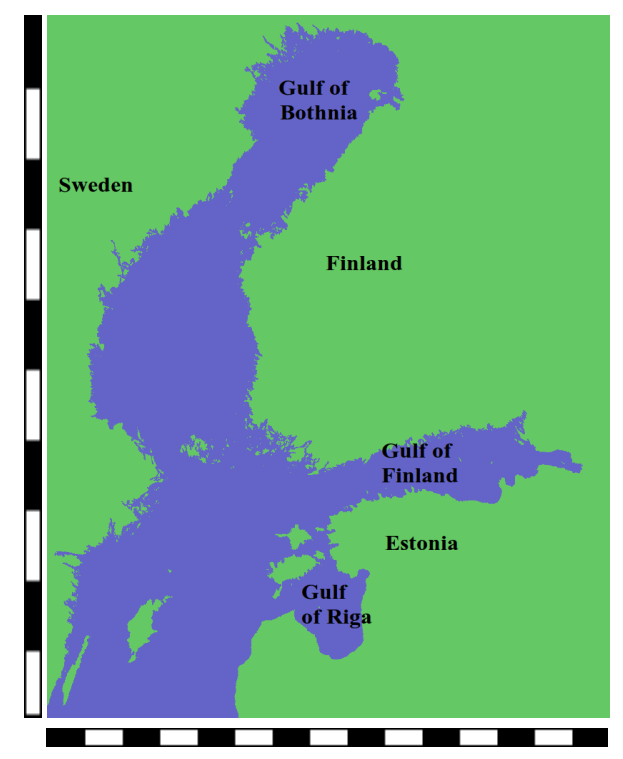

Figure 1. The Baltic Sea study area. In the image scales the interval is $1^{\circ}$ in both latitude and longitude; the upper left corner is located at $66^{\circ} \mathrm{N}, 16^{\circ} \mathrm{E}$, and the lower right corner is located at $56^{\circ} \mathrm{N}, 31^{\circ} \mathrm{E}$.

kilometres) remain stable and typically have a high concentration throughout the winter. Winter 2013-2014 was a mild ice winter in the Baltic, and the air temperature also varied from cold to warm (above zero) many times during the winter.

The radiometer data were AMSR-2 radiometer level 1R brightness temperature data (Maeda, 2013). The SAR data were dual-polarized (HH/HV polarization combination) RADARSAT-2 ScanSAR Wide mode data. In this study we only utilized the HH channel of the SAR data. The SAR mosaics were georectified to Mercator projection, and an incidence angle correction was applied to each SAR image included in the mosaic. The ice concentration estimates were also produced in Mercator projection, mapped and resampled to the resolution of $500 \mathrm{~m}$, which corresponds to the projection and resolution of our SAR mosaics. In the projection, we used the WGS84 datum, and the reference latitude (true scale latitude) was $61^{\circ} 40^{\prime} \mathrm{N}$. The data set was divided into a training data set, consisting of daily SAR mosaics and AMSR-2 brightness temperature mosaics from 23 January to 1 February 2014, and a test data set, consisting of daily SAR mosaics and the corresponding daily AMSR-2 brightness temperature mosaics from 2 to 11 February 2014. Both data sets included 10 SAR mosaics and the corresponding daily AMSR-2 brightness temperature images.

The study period also ranged from 23 January to 11 February 2014. During this period the air temperature varied quite a lot; the lowest hourly mean air temperatures during the test period at the Tankar coastal weather station (location $63.95^{\circ} \mathrm{N}, 22.84^{\circ} \mathrm{E}$ ) were around $-17^{\circ} \mathrm{C}$ and the highest around $+3^{\circ} \mathrm{C}$. The weather conditions during the training 
period varied; there was a short cold period (1-2 days), then a warmer period and then colder again. During the test period, there were two warmer periods and one colder period (not as cold as the one short cold period during the training period with the lowest temperature of $-17^{\circ} \mathrm{C}$ ). There were also cases of wet snow over the sea ice and dry snow over the sea ice during both the training and the test period.

We also performed tests for two of our test area image mosaics in the Kara and Barents seas. In this Arctic test area the projection was polar stereographic with the WGS84 datum. The mid-longitude was $55^{\circ} \mathrm{E}$ and the true-scale latitude $70^{\circ} \mathrm{N}$. The latitudes in the area range from about 65 to $85^{\circ} \mathrm{N}$ and longitudes from 0 to $90^{\circ} \mathrm{E}$. The ice and weather conditions in this Arctic area are different from the Baltic Sea. Typically the weather during the winter months is colder, usually clearly below $0{ }^{\circ} \mathrm{C}$, and the snow cover is typically wet only during the melting period starting in May-June. Also, in this Arctic test area, the ice concentrations vary because the ice is moving in most parts of the Arctic test area. Due to the colder temperatures less ice melting occurs during wintertime. In the Baltic, it is typical that some areas melt and refreeze during the wintertime due to rapid temperature changes.

\section{Ice concentration estimation algorithm}

\subsection{SAR Processing}

The SAR images were processed according to our standard procedure: first, they were calibrated and rectified to Mercator projection; then an incidence angle correction was applied according to Makynen et al. (2002). After this, daily SAR mosaics were computed by remapping the SAR images onto the study area such that newer data were always overlaid over older data, producing mosaics with the newest SAR data in each mosaic grid cell or pixel. The resolution of the SAR mosaics was $500 \mathrm{~m}$. After this a segmentation step was performed for the daily mosaic. Here we have used an MRF-based segmentation adapted from Kato et al. (1992) and Berthod et al. (1996), but in practice any feasible segmentation, such as ICM (iterated conditional modes) (Besag, 1986) or even $K$ means (MacQueen, 1967), can be used with rather similar results. The SAR segments smaller than 100 pixels (corresponding to an area of $25 \mathrm{~km}^{2}$ ) were combined with the adjacent larger segments with the closest $\mathrm{HH}$ backscattering value (by an iterative process). The incidence angle correction has been designed for sea ice, and, in general, it does not work over open-water areas, where the SAR backscattering is dependent on the water surface roughness, i.e. waves. Wave conditions can change rapidly depending on the winds, and a good incidence angle correction over open water would require reliable wave magnitude and direction information (i.e. a two-dimensional wave spectrum). Due to the varying wave conditions, the backscattering from open water in different SAR images can vary significantly.
This can naturally be seen in SAR mosaics and affects the SAR segmentation in these areas. However, this effect is not a problem in, e.g., concentration estimation or segment classification as long as open water and segments with sea ice are separated by the segmentation. We have tested our segmentation algorithm for SAR data of two whole Baltic Sea ice seasons, including all possible wind and temperature conditions, and according to our experience the results are very reliable and large ice areas and large open-water areas are separated almost in every case. The possible errors may occur in warm conditions with very wet snow or water over ice. Even in these cases we get reasonable ice concentration estimates for the segments possibly containing large areas of both open water and sea ice, but then we do not necessarily have the information where within the (possibly large) segment sea ice and open water (ice edge) are located. We have also tried performing this segmentation using so-called principal component (PC) images from the two channels of dual-polarized SAR imagery. PC images consist of the first principal component computed of the two channels. According to our experience this has not improved the segmentation significantly with regard to separating between sea ice and open water - i.e. having sea ice and open water in different segments - when compared to visual interpretation. PC images, however, improve the ice type segmentation in some cases (e.g. by better distinguishing between instances of deformed ice). With a good incidence angle correction the SAR frame boundaries are not visible in the ice-covered areas and the ice segments correspond to natural ice areas. Separating between natural open-water segments is not possible because the wave conditions (surface roughness) change on much faster timescales and because of backscattering, but on the other hand in our application we are not interested in classifying open water, e.g., based on surface roughness in more detail, but just in locating open-water segments and assigning zero ice concentration to these segments.

\subsection{AMSR-2 processing}

The AMSR-2 brightness temperature data were processed into daily mosaics similarly to SAR mosaicking; i.e. the new data were always written over older data. This data were presented at a $10 \mathrm{~km}$ resolution. The brightness temperatures used in this study ranged from $18.7,23.8,36.5$ and $89.0 \mathrm{GHz}$, here denoted by $18,23,36$ and $89 \mathrm{GHz}$ channels, respectively. All the AMSR-2 channels have both $\mathrm{H}$ and $\mathrm{V}$ polarizations. From the daily brightness temperature mosaic data, all the polarization ratios (PRs) and gradient ratios (GRs) were derived according to the following equations:

$\operatorname{PR}(f)=\frac{T_{\mathrm{B}}(f, V)-T_{\mathrm{B}}(f, H)}{T_{\mathrm{B}}(f, V)+T_{\mathrm{B}}(f, H)}$

and

$\mathrm{GR}(f 1, f 2, p)=\frac{T_{\mathrm{B}}(f 1, p)-T_{\mathrm{B}}(f 2, p)}{T_{\mathrm{B}}(f 1, p)+T_{\mathrm{B}}(f 2, p)}$. 
By computing the polarization ratios for all the four frequencies, denoted by $f$, and the pairwise gradient ratios for all the four frequencies and for the two polarizations, denoted by $p$, we get 4 polarization ratios and 12 gradient ratios at each grid cell. These are then used in the concentration estimation. The advantage of using polarization ratios is that they reduce the dependence on temperature; see, e.g., Steffen et al. (1992). If the brightness temperatures were used, then also temperature estimates (e.g. from a numerical weather prediction model) or measurements at each grid point would be necessary for reliable ice concentration estimation.

A land mask, which was derived from the GSHHG (Global Self-Consistent Hierarchical, High-resolution Geography database from the National Oceanic and Atmospheric Administration, NOAA) coastline data (Wessel and Smith, 1996), was applied to the brightness temperatures at their original (low) resolution. Because mixed land-sea pixels complicate the computation, they are either omitted or handled differently in the computation (Maass and Kaleschke, 2010). In this study the mixed land-sea pixels were omitted by our land masking. After this the brightness temperature values were extrapolated to cover a larger area overlapping the land areas. This technique was used to avoid resampling artifacts in the next phase and to guarantee a concentration estimate for each SAR segment. After this the brightness temperature grids were up-sampled to the SAR mosaic resolution $(500 \mathrm{~m})$ and a land masking at this high resolution was applied. This approach enables us to compute ice concentration estimates for the coastal SAR segments also.

We did not apply any explicit weather filtering. Typically, weather filtering is based on ratios of channels sensitive to enhanced environment emission over open water. The NASA Team, bootstrap and ASI algorithms use gradient ratios between 23.8 and 18.7 , and 36.5 and $18.7 \mathrm{GHz}$, respectively, in their weather filters. All these ratios are inputs to our neural network algorithm. If we assume that we have a representative training data set including enough samples of all the possible weather conditions, then the neural network should also be able to take the weather conditions into account. Our training data set only covered a relatively short period and was not representative in this sense, so it is expected that some estimation errors due to weather may occur.

\subsection{Combining brightness temperatures and SAR}

After SAR segmentation we computed the mode of each polarization ratio and gradient ratio for each SAR segment. The mode was computed from up-sampled AMSR-2 polarization ratio and gradient ratio grids. Mode was selected as a metric because it is more robust with respect to outliers, e.g. due to weather effects or variability in surface conditions. The concentration estimation for each SAR segment was based on these segment-wise modes of polarization and gradient ratios as MLP inputs. In this way we are able to produce an ice concentration estimate in the SAR mosaic resolution. The boundaries of different ice concentration areas corresponding to SAR segments are produced in the SAR resolution. Typically at the boundaries of ice and open water there are mixed ice-water pixels and the concentration estimates at the edges are blurred. Using the SAR resolution reduces this blurring at the edges and produces sharper boundaries at the edges. Naturally the method is unable to extract smaller details than defined by the AMSR-2 resolution, but as concentration is a function of the resolution we still get reasonable concentration estimates over these areas, too.

\subsection{Concentration estimation}

The ice concentration estimation is based on an MLP neural network. The neural network was trained using the error backpropagation algorithm (Haykin, 1999). Furthermore, the neural network was trained using the FMI (Finnish Meteorological Institute) gridded ice charts as its reference input. The hidden layer nonlinearities were implemented using the hyperbolic tangent (tanh) function. The single unit or artificial neuron of the output layer corresponding to the one MLP output (the estimated ice concentration) was linear. Feed-forward neural networks, such as MLP, with a single hidden layer of sigmoidal units are capable of approximating any continuous multivariate function to any desired degree of accuracy (Hornik, 1989).

The output $y_{i}$ of each MLP unit with index $i$ is computed as

$y_{i}=f_{i}\left(\sum_{j} W_{i j} y_{j}\right)$,

where $f_{i}$ is the activation function of the unit (also known as a node or a neuron) $i$. At the hidden layer, the mapping is $f_{i}(x)=\tanh (x)$, and at the output layer the mapping is linear, i.e. $f_{\mathrm{o}}(x)=x$. The weights $W_{i j}$ are related to each input $y_{j}$. The inputs $y_{j}$ are also outputs from the previous MLP layer (or inputs at the input layer). In the training phase the outputs are first computed in the forward direction, and then the error is propagated back from the MLP output layer, i.e. starting from the error between the ice concentration estimate given by the MLP and the desired ice concentration defined by the training data, towards the input layer. A detailed derivation and presentation of the error backpropagation learning rule can be found, e.g., in Haykin (1999). In our case the only output is the ice concentration, and thus our MLP has only one output unit. In the training phase, the weights $W_{i j}$ are updated towards the negative gradient of the error function at each node. To include a constant term $\left(W_{k 0}\right)$, 1.0 is also input into each MLP unit.

The MLP architecture used was 17-20-1, i.e. 16 inputs corresponding to the 4 polarization ratios, 12 gradient ratios and the constant term (input 1.0), 20 hidden-layer nonlinear units and 1 linear output layer unit, whose output is the ice concentration estimate. A schematic diagram of the MLP used 


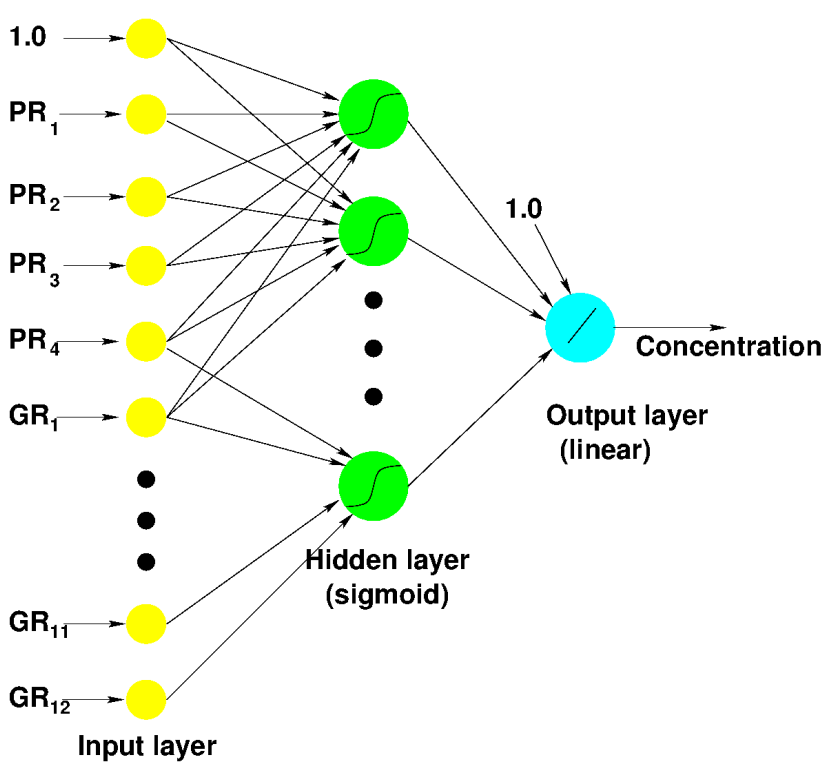

Figure 2. A schematic diagram of the MLP architecture used; there are 17 input layer units (including the constant term 1.0, 20 hiddenlayer sigmoid units and one linear output layer unit). For clarity not all the connections have been drawn in the figure.

is shown in Fig. 2. The number of the hidden-layer units was determined experimentally: starting from two hidden-layer neurons, then iteratively increasing their number and performing the training until the training error did not decrease notably any more by adding more hidden-layer neurons. Because the algorithm makes the estimation segment-wise, not pixel-wise, the estimation is rather fast (in practice it can be run for one mosaic image in a time range of a few seconds to a few tens of seconds on a desktop personal computer, depending on the image and computing power of the computer). We used the so-called epoch training (i.e. the whole training data set is iteratively fed through the MLP in a random order) and each iteration corresponds to one epoch. In the following equations the epochs are indicated by the time variable $t$, which is an integer number starting from 1 . The learning rate parameter $\mu$ is adaptive. At the first epoch the learning rate is set to 0.005 and it is adjusted after each epoch $t$ depending on the total MLP error $E$ :

$\begin{array}{ll}\mu(t+1)=1.05 \mu(t) \quad \text { if } \quad & E(t)<E(t-1), \\ \mu(t+1)=0.70 \mu(t) & \text { if } \quad E(t) \geq E(t-1) .\end{array}$

The number of training epochs used was 20000 . The MLP coefficients $W_{i j}$ were initialized randomly, and the MLP coefficients corresponding to the minimum MLP total error $E$ among 40 training runs were selected as the final MLP parameters, which are then used in the ice concentration estimation. This approach guarantees the exclusion of a poor selection of the initial MLP weight coefficient configuration.

\section{Evaluation}

We evaluated the algorithm results by comparing them to the FMI digitized ice chart grids, which have a nominal resolution of $1 \mathrm{~km}$, and to the high-resolution $(3.125 \mathrm{~km})$ UH ASI AMSR-2 algorithm (Beitsch et al., 2013, 2014) results. We also made a visual comparison to the results of the AMSR-2 bootstrap algorithm ice concentrations provided by JAXA (Japan Aerospace eXploration Agency) (Maeda, 2013; JAXA, 2013), which have a resolution of $10 \mathrm{~km}$. The bootstrap algorithm uses the AMSR-2 channels of $36.5 \mathrm{GHz} \mathrm{V}$ (vertical), $36.5 \mathrm{GHz} \mathrm{H}$ (horizontal), $18.7 \mathrm{GHz} \mathrm{V}$ and $23.8 \mathrm{GHz} \mathrm{V}$ for the weather filter. We also performed some comparisons over an Arctic Sea test area (Kara and Barents seas), over which we made daily SAR mosaics. We used the same training data as used for the Baltic Sea, and the comparison to the AMSR-2 level 2 concentration results showed good agreement based on visual judgment: the highand low-concentration areas in general corresponded to each other.

A comparison between SAR-based ice concentration and reference data was made by using three error measures: $L_{1}$ error $E_{L_{1}}$; the signed $L_{1}$ error $E_{\mathrm{sgn}}$ (describing the bias); and root mean square (rms) error $E_{\mathrm{RMS}}$ :

$$
\begin{aligned}
& E_{L_{1}}=\frac{1}{N_{s}} \sum_{i=1}^{N_{s}}\left|C_{i}^{\mathrm{est}}-C_{i}^{\mathrm{ref}}\right|, \\
& E_{\mathrm{sgn}}=\frac{1}{N_{s}} \sum_{i=1}^{N_{s}}\left(C_{i}^{\mathrm{est}}-C_{i}^{\mathrm{ref}}\right), \\
& E_{\mathrm{RMS}}=\sqrt{\frac{1}{N_{s}} \sum_{i=1}^{N_{s}}\left(C_{i}^{\mathrm{est}}-C_{i}^{\mathrm{ref}}\right)^{2} .}
\end{aligned}
$$

$N_{s}$ is the number of pixels or grid cells computed over the whole ice concentration estimate grid area, $C_{i}^{\text {est }}$ is the estimated concentration and $C_{i}^{\text {ref }}$ is the reference concentration at each pixel indicated by the index $i$. The error measures were computed over the SAR image mosaic area (with a grid cell spacing of $500 \mathrm{~m}$ ), and the reference data were sampled at the same resolution by using bilinear sampling. The comparisons were also grid-cell-based comparisons in the SAR mosaic resolution of $500 \mathrm{~m} . E_{L_{1}}(\geq 0)$ describes the mean absolute error, and $E_{\mathrm{sgn}}$ describes the bias or systematic error. If $E_{\mathrm{sgn}}<0$, the algorithm underestimates, and if $E_{\mathrm{sgn}}>0$, the algorithm overestimates the ice concentration compared to the reference data. The RMSE represents the sample standard deviation of the differences between the estimated values and the reference values.

The comparison results can be seen in Table 1; the errors are given in percentage points. We also provide the standard deviations for the error measures, describing the variations between the images. For the training data set, the ice concentration is slightly overestimated (2.95 percentage points), and, for the test data set, it is slightly underestimated (3.91 
Table 1. Errors for our algorithm results compared to FMI ice charts for the training and test data sets. The latitude range of the area is $56-66^{\circ} \mathrm{N}$ and the longitude range $16-31^{\circ} \mathrm{E}$; the results were computed for data rectified to Mercator projection with a resolution of $500 \mathrm{~m}$. For both the data sets (training and test sets) the number of scenes $N=10$.

\begin{tabular}{lrrr}
\hline Measure & Signed error & $L_{1}$ error & RMSE \\
\hline \multicolumn{2}{l}{ Training data set } \\
& & & \\
\hline Error & 2.95 & 8.67 & 19.98 \\
SD & 3.33 & 2.02 & 2.42 \\
\hline Test data set & & & \\
\hline Error & -3.91 & 8.66 & 21.28 \\
SD & 2.67 & 1.98 & 3.29 \\
\hline
\end{tabular}

percentage points); the mean $L_{1}$ error for the training and test data sets is practically the same, about 8.7 percentage points. The standard deviations of the errors are relatively low for all the error measures, indicating that the errors are quite similar for all the data. Two examples of the estimation result, the corresponding FMI ice chart concentrations and the corresponding bootstrap algorithm concentration estimates can be seen in Figs. 3 and 4. In the SAR mosaic and segmentation result (see Fig. 3), we can see that the open-water areas produce different backscattering, depending on the prevailing wave conditions, and this can be seen as clear SAR frame boundaries in the open-water areas of the SAR image mosaic and as many separate open-water segments in the segmentation result. However, in the ice-covered areas the SAR frame boundaries are not visible, indicating that the incidence angle correction for sea ice has been successful. The ice edge has been sketched as a red line in the SAR mosaic of Fig. 3a; the ice-covered area is in the northern and eastern side of the ice edge line. This indicates that the ice segments can be identified correctly over SAR frame boundaries in the mosaic, enabling computation of statistics and classification of the ice classes, and they are also separated from open-water segments. In Figs. 3 and 4, we can, for example, see that the new algorithm is unable to capture parts of the ice-covered areas in the northern parts of the Gulf of Finland (latitude around $\left.60^{\circ} \mathrm{N}\right)$. These areas are separated from open water by the SAR segmentation, but, as they are located near the coast, they are problematic for a radiometer at a low resolution (mixed pixels); for this reason the near-coast radiometer pixels are omitted. However, after this, to be able to assign a concentration estimate to each SAR segment, the radiometer brightness temperatures are extrapolated as described in Sect. 3.2.

The estimates for the ice zones in the Gulf of Riga (located in the southern part of the images, latitude around $58^{\circ} \mathrm{N}$, longitude $24^{\circ} \mathrm{E}$ ) correspond to the ice chart concentration much better than the bootstrap algorithm result in both cases

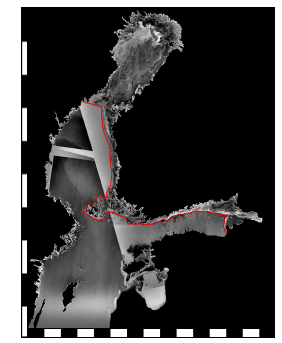

(a)

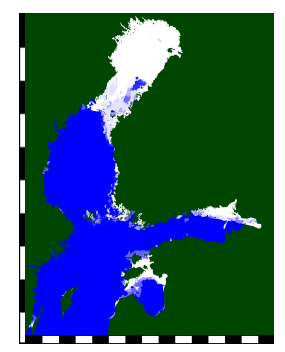

(d)

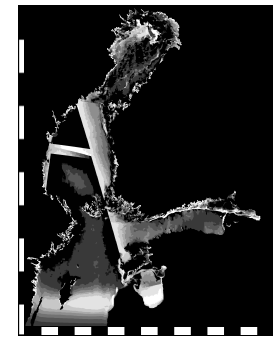

(b)

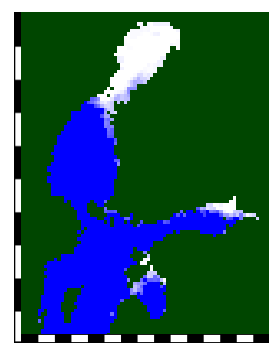

(e)

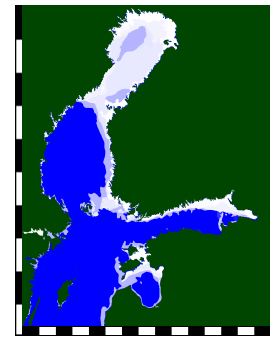

(c)

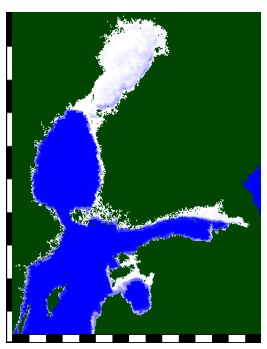

(f)

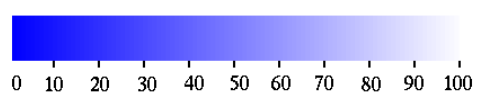

Figure 3. SAR image mosaic of 2 February 2014, ${ }^{\circ}$ MDA (a), the ice edge has been indicated by the red line; segmentation result (b); FMI ice chart grid ice concentration (c); ice concentration estimate using our algorithm (d); AMSR-2 bootstrap algorithm ice concentration given at a $10 \mathrm{~km}$ resolution (e); ASI ice concentration (f). In the image scales the interval is $1^{\circ}$ in both latitude and longitude; the upper left corner is located at $66^{\circ} \mathrm{N}, 16^{\circ} \mathrm{E}$, and the lower right corner is located at $56^{\circ} \mathrm{N}, 31^{\circ} \mathrm{E}$.

of 2 February and 11 February, presented in Figs. 3 and 4. Also, for the 11 February case in Fig. 4, our algorithm gives high concentrations similar to the ice chart in the mid-parts of the Gulf of Bothnia (northern parts of the area, a latitude about $63.5^{\circ} \mathrm{N}$, longitude about $20^{\circ} \mathrm{E}$ ). The bootstrap algorithm seems to underestimate the concentration in these areas. In Fig. 5 we can see a detail near the ice edge produced by our algorithm, the bootstrap algorithm and ASI algorithm. The different resolution of the different products can be seen in the details along the edge. In Figs. 6 and 7 we show the differences between the FMI ice chart and our algorithm result, as well as a similar comparison to the UH ASI algorithm results. The ASI concentration estimates are also included in Figs. 3 and 4. This version utilizes the full-resolution AMSR2 data, and it can even capture the ice-covered zone in the northern Gulf of Finland. However, in the coastal zone the ASI algorithm seems to overestimate the ice concentration. This can be seen in both Figs. 3 and 4 as non-zero concentrations, for example along the southern Swedish coast, where the other products or FMI ice charts do not indicate ice. Our new algorithm produces lower concentration estimates in the coastal zone. The largest differences between the FMI ice chart concentration and ASI concentration occur at the ice 


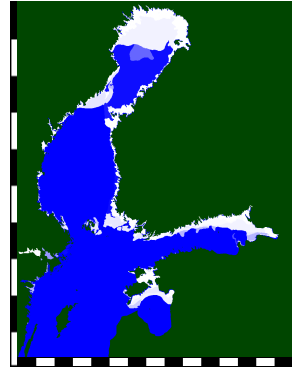

(a)

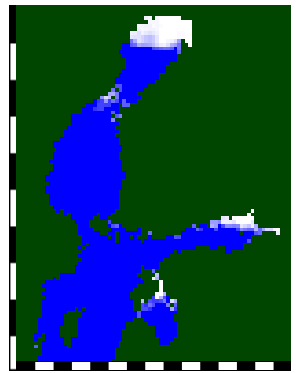

(c)

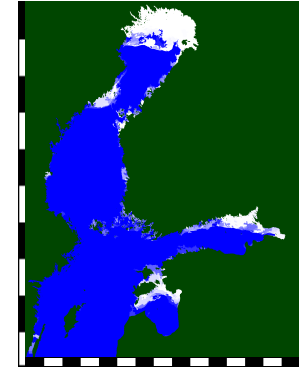

(b)

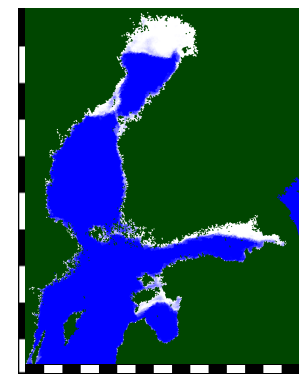

(d)

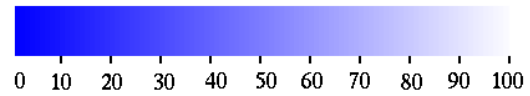

Figure 4. FMI ice chart concentration grid for 11 February 2014 (a); ice concentration estimate using our algorithm (b); AMSR-2 bootstrap algorithm ice concentration (c); and ASI ice concentration (d). In the image scales the interval is $1^{\circ}$ in both latitude and longitude; the upper left corner is located at $66^{\circ} \mathrm{N}, 16^{\circ} \mathrm{E}$, and the lower right corner is located at $56^{\circ} \mathrm{N}, 31^{\circ} \mathrm{E}$.

boundaries (ice edge), which have higher precision in FMI ice charts as well as in our combined radiometer-SAR product. The numerical results of this comparison are given in Tables 2 and 3. We also performed this comparison in the ASI products resolution, and the error measures were 2.64, 10.80 and 22.95 percentage points for the signed error, $L_{1}$ error and RMSE, respectively.

In some open-water areas in the western parts of the Arctic test area mosaic over the Kara and Barents seas, some overestimations of the ice concentration occur, e.g. the openwater area in the mid-left part of the images of Figs. $8 \mathrm{~b}$ and 9. around an approximate coordinate location $(x, y)=(200$, $800)$. These are probably due to different weather conditions and open-water signatures than in the Baltic Sea (training data set) and would probably be corrected by also including data from the Arctic in the training phase. Arctic training data were not used in this study, because we did not have Arctic high-resolution ice chart concentrations available. The Arctic test was made just to see whether we can get reasonable results using the Baltic Sea training data. Also, in the Arctic, data sources other than ice charts must be used for training; these could be, for example, ASI or some other radiometerbased concentration results.

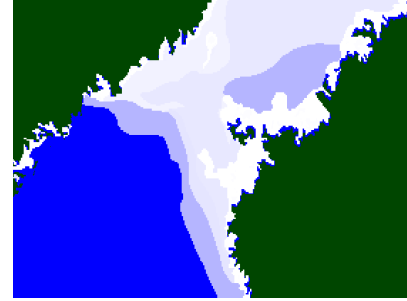

(a)

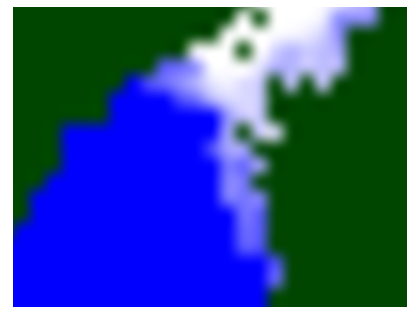

(c)

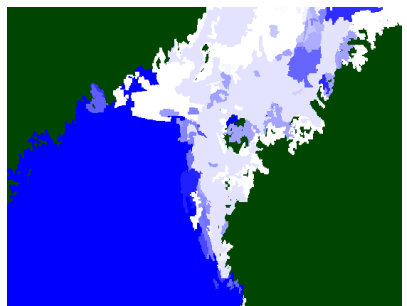

(b)

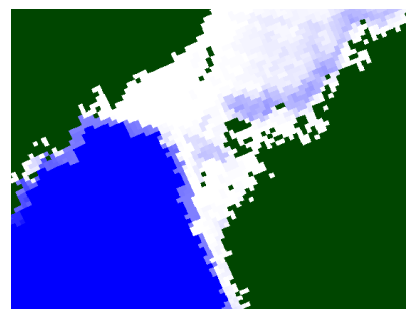

(d)
Figure 5. A detail of the 2 February 2014 concentration estimates near the ice edge. FMI ice chart concentration (a); our algorithm result (b); bootstrap ice concentration (c); and ASI ice concentration (d). In the image scales the interval is $1^{\circ}$ in both latitude and longitude; the upper left corner is located at $66^{\circ} \mathrm{N}, 16^{\circ} \mathrm{E}$, and the lower right corner is located at $56^{\circ} \mathrm{N}, 31^{\circ} \mathrm{E}$.

In general it can be said that our algorithm results are slightly better than the ASI results when compared to the FMI ice chart grids. This was an expectable result because we have used FMI ice charts in training of our algorithm. The error measures for the ASI algorithm results compared to FMI ice chart grid results are a bit larger, as shown in Tables 2 and 3 .

Some comparisons between radiometer ice concentration products and other radiometer-based ice concentration products as well as visual ice concentration observation onboard a ship, e.g. in Spreen et al. (2008), and visual/infrared (VIS / IR) earth-observation data, e.g. in Cavalieri et al. (2006), have been made. The VIS / IR comparison requires clear-sky conditions or a reliable cloud mask. The correlation between the visual estimates and the ASI algorithm concentrations in Spreen et al. (2008) was around 0.8; in the intercomparison between ASI, the NASA Team 2 algorithm and the bootstrap algorithm the RMSE ranged from 7.2 to 10.8 and bias (signed error) ranged from -2.0 to 1.7. All the compared algorithms used AMSR-E (Advanced Microwave Scanning Radiometer Earth Observing System) radiometer data as their input. In Cavalieri et al. (2006) a comparison between Landsat-7-based ice concentrations and NASA Team 2 algorithm concentrations was made for some images, and RMSEs were in the range of 1-7 and biases in the range of $0-3$. However, these results cannot directly be compared to our error measures because the comparisons have been made between different quantities. 
Table 2. Errors for our algorithm results compared to ASI ice concentrations for the test data set and for two Arctic Ocean cases (corresponding to two daily SAR mosaics). The results were computed for data rectified to polar stereographic projection with a resolution of $500 \mathrm{~m}$.

\begin{tabular}{lrrr}
\hline Measure & Signed error & $L_{1}$ error & RMSE \\
\hline \multicolumn{4}{l}{ Test data set (Baltic) } \\
\hline Error & -1.23 & 11.15 & 25.19 \\
SD & 7.84 & 4.15 & 5.35 \\
\hline Arctic Ocean test cases & & \\
\hline Error & -3.29 & 6.68 & 16.85 \\
SD & 5.56 & 4.20 & 5.98 \\
\hline
\end{tabular}

Table 3. Errors for the comparison between FMI ice charts and the ASI results. The same Mercator projection as in the other Baltic Sea comparisons was used.

\begin{tabular}{lrrr}
\hline Measure & Signed error & $L_{1}$ error & RMSE \\
\hline \multicolumn{2}{l}{ Test data set (Baltic) } & & \\
\hline Error & 5.38 & 11.81 & 27.30 \\
SD & 4.31 & 2.54 & 3.28 \\
\hline
\end{tabular}

The comparisons to the bootstrap ice concentrations provide by JAXA were visual evaluations of the differences. The results of our algorithm correspond to the results of the bootstrap algorithm quite well, based on a general visual overview, but there are still some visible differences, especially in details due to different resolutions of the products. However, our algorithm also gives ice estimates for the coastal areas and at the boundaries of different ice concentration zones in the SAR mosaic resolution. The results of our algorithm and bootstrap ice concentrations are also seen in Figs. 3 and 4. We did not make numerical comparisons, because of the significantly different resolutions; in this case the effect of the resolution would probably have contributed more to the computed error than the actual error. Some rough estimates of the effect of the different resolutions to the concentration estimation error can be found, e.g., in Karvonen (2014). To improve the performance, more training data both from the Arctic and the Baltic Sea including different weather and ice conditions would be required to make a representative data set of the possible weather and ice conditions. Also, studies on using the radiometer data sampled at a higher resolution (similar to ASI) will be used to improve the algorithm performance in the future.

We also made a visual comparison for two ice concentration estimations over an Arctic Ocean test area in the Barents and Kara seas. Also, in these areas, the bootstrap algorithm concentrations and concentrations produced by our algorithm were in good agreement - as an example, see Fig. 8

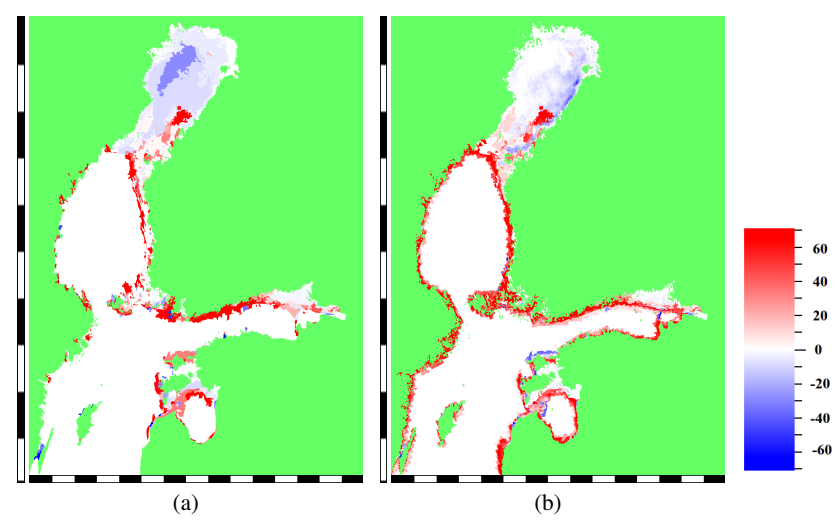

Figure 6. Difference between the algorithm result and FMI ice chart concentration for 2 February 2014 (a) and between the algorithm result and ASI concentration (b). Overestimation produced by our algorithm with respect to the reference data is indicated by the blue tones (negative difference values) and underestimation by red (positive values). In the image scales the interval is $1^{\circ}$ in both latitude and longitude; the upper left corner is located at $66^{\circ} \mathrm{N}, 16^{\circ} \mathrm{E}$, and the lower right corner is located at $56^{\circ} \mathrm{N}, 31^{\circ} \mathrm{E}$.

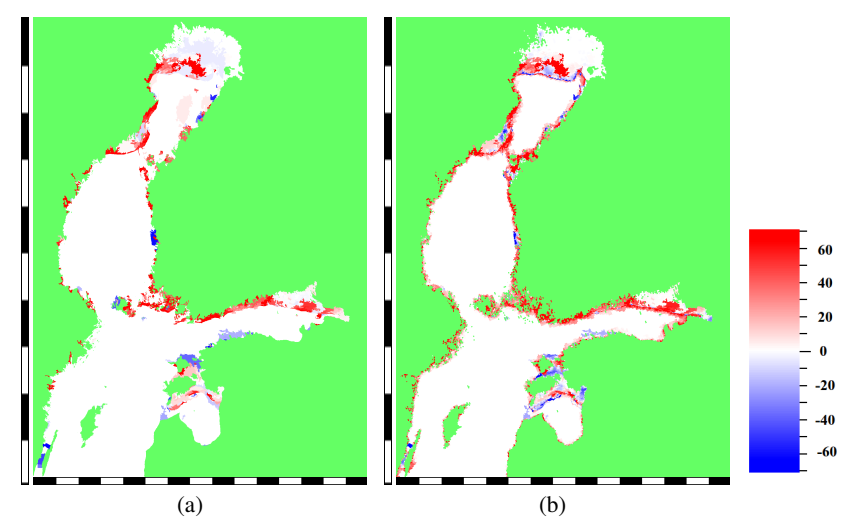

Figure 7. Difference between our algorithm result and FMI ice chart concentration for 11 February 2014 (a) and between our result and ASI concentration (b). Overestimation produced by our algorithm with respect to the reference data is indicated by the blue tones (negative difference values) and underestimation by red (positive values). In the image scales the interval is $1^{\circ}$ in both latitude and longitude; the upper left corner is located at $66^{\circ} \mathrm{N}, 16^{\circ} \mathrm{E}$, and the lower right corner is located at $56^{\circ} \mathrm{N}, 31^{\circ} \mathrm{E}$.

- even though the training was performed with a rather limited set of Baltic Sea data. We also compared our algorithm results to the ASI high-resolution results over this area for our two test images; the resulting difference map for the case shown in Fig. 8 is shown in Fig. 9. For this comparison we also give the error statistics in Table 2, but due to a very small Arctic data set these values are not very confident.

We did not find a significant difference in the performance of our algorithm and the ASI algorithm in different concentration ranges. The error compared to FMI ice charts was similar, but in some cases the errors were made at different 


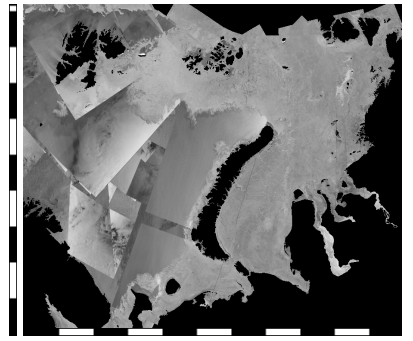

(a)

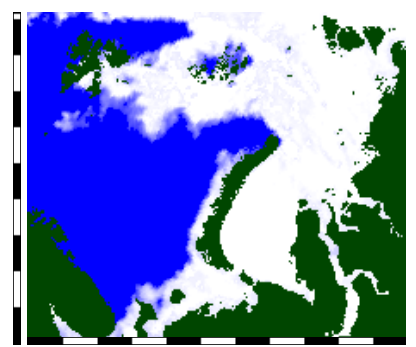

(c)

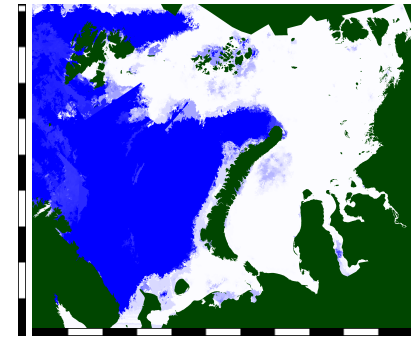

(b)

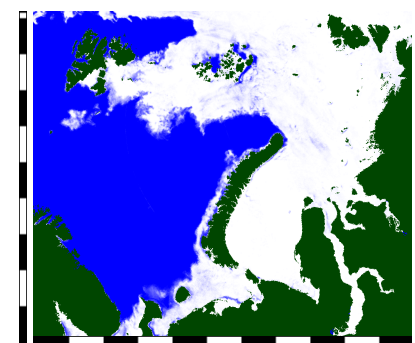

(d)

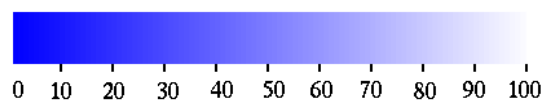

Figure 8. An example of the Arctic Ocean ice concentration estimation: the SAR mosaic of 2 February 2014 (a); ice concentration estimate based on our algorithm (b); the AMSR-2 level 2 ice concentration product (c); and ASI ice concentration product (d). Some parts of the area are not covered by the SAR imagery in the SAR mosaic (these areas are indicated by black tone in the SAR mosaic as is the land mask area. Areas not covered by SAR imagery appear in the upper part of the mosaic area and also in the lower right part of the mosaic) and the concentration of our product is only given in the area of the SAR mosaic cover. The image scale interval is $200 \mathrm{~km}$; the sides of the whole image are $2200 \mathrm{~km}$ ( $x$ direction, horizontal) and $1850 \mathrm{~km}$ ( $y$ direction, vertical) long. The origin of the coordinate system referred to in the text is at the lower left corner.

locations. The concentration distributions for our algorithm have less mid-range concentration values compared to the bootstrap algorithm and the ASI algorithm, and the difference is larger for the bootstrap algorithm. This is due to the reduced blurring at the (open-water and ice) edges. Because of its high resolution, less edge blurring is generated by our algorithm.

\section{Discussion}

The results from the comparison to FMI ice charts were better than, for example, those reported for dual-polarized SAR data in Karvonen (2014). The best concentration estimates can very likely be achieved by combining SAR data and using radiometer data as a background value, such as has been done with the ice thickness values in Karvonen et al. (2008), where modelled sea ice thickness is used as background

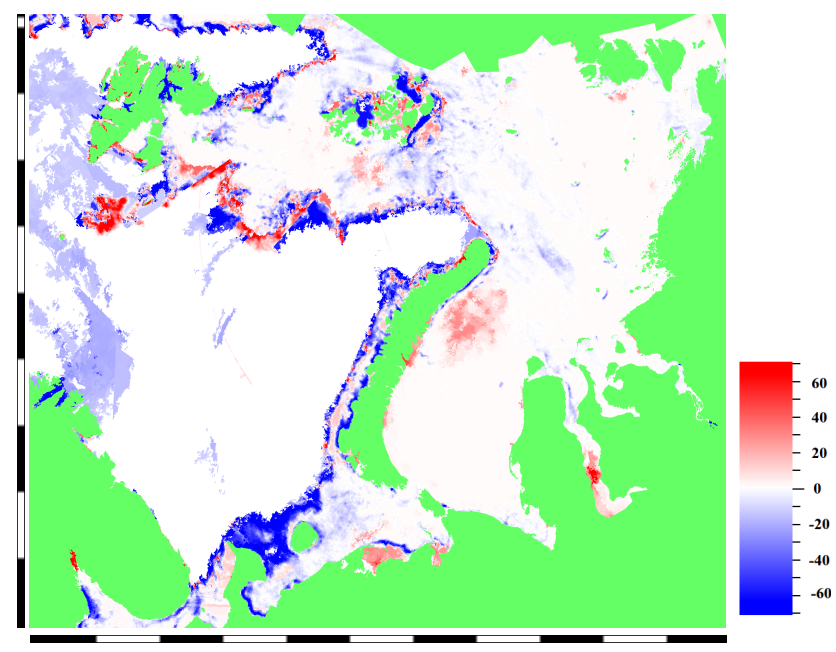

Figure 9. The difference between the FMI algorithm concentration and ASI ice concentration for the Arctic study area, 2 February 2014. The areas where our algorithm indicates higher concentration than ASI appear as blue, and areas where our algorithm indicates lower concentration appear as red. The image scale interval is $200 \mathrm{~km}$; the sides of the whole image are $2200 \mathrm{~km}$ ( $x$ direction, horizontal) and $1850 \mathrm{~km}$ ( $y$ direction, vertical) long.

information and SAR imagery is used to improve the resolution of the estimates. The methodology may differ, but the basic idea is to enhance the background information based on SAR data to yield more precise estimates in a higher resolution compared to the background data resolution.

The training was performed using the polarization ratios and gradient ratios, because they provide a more stable basis for the ice concentration estimation with respect to the temperature changes. We also studied estimation by directly feeding the brightness temperatures into the MLP, but the MLP convergence was slower and estimation results worse with this approach. The reason for this behaviour was obviously that to be able to also model the temperature dependence a representative training data set describing a wide range of temperature conditions would be required, and our training data set of 10-day mosaics was too small for that purpose. However, it seems to be large enough when using ratios instead of brightness temperatures directly. We tested this by training our MLP by using the training data set brightness temperatures as inputs. The convergence of the algorithm was very slow (no good convergence even after 100000 epochs), and, after training the MLP, the estimation results were poor even for the test set; the mean errors were up to 40 percentage points for many image mosaics. When using polarization and gradient ratios as inputs, the convergence was faster and the error measures for the training data set reasonable. We believe even better results can be achieved by using an extensive representative training data set covering all possible ice types and weather conditions. However, already this experiment clearly indicates the potential of the 
methodology for providing high-resolution operational ice concentration estimates.

We have used the resampled AMSR-2 L1R (Level 1 Resampled) data which have a resolution of about $10 \mathrm{~km}$. We can see that the ASI algorithm utilizing the full AMSR-2 resolution (full resolution of the higher-frequency channels) is capable of distinguishing details which are not all visible in the L1R data. It would be desirable to use the best possible resolution in the future operational algorithm, and we are going to adapt our MLP algorithm to work with the highprecision data also. After this high-resolution radiometer estimate we can still refine the segment boundaries based on SAR data to yield improved precision. Actually, all we need to do is to train the algorithm with suitably sampled highresolution data for a representative training data set; after this it is capable of utilizing the full resolution.

The comparison results show that our algorithm estimates are closer to the FMI ice charts than the other two reference algorithms (bootstrap and ASI). This was an expected result, because the FMI ice charts are mainly based on visual interpretation of SAR data, and FMI ice charts and SAR data have been used in the training; thus our algorithm has in a way been adapted to the FMI ice charts.

We were also surprised by the good estimation results in the Arctic test area because the training data were from a relatively short period and from the Baltic Sea area. This can at least be partly explained by the fact that, in our Arctic test area, mainly only seasonal sea ice exists; only a little (less than $1 \%$ of the whole image area) multi-year ice can appear in the northeastern parts of the area. In areas with more multiyear ice, the algorithm should be trained with similar data for reliable ice concentration estimates. Because in the Arctic, we do not have digitized ice charts at our disposal, we should use other data sources for the training. One such data source could be the results of other radiometer ice concentration algorithms, such as the bootstrap and ASI algorithms used as reference data here, refined by SAR imagery as presented in Sect. 3.3.

The advantage of the MLP approach is that it is not necessary to define parameters related to the brightness temperatures or ratios; all we need is a representative training data set to train the MLP. The disadvantage is that the actual nonlinear mapping from the input parameters to ice concentration remains unknown. The mapping is naturally described by the MLP weights and MLP structure, but the physical relationship between inputs and outputs remains unclear.

The novel method results in improved accuracy of the boundaries of the ice zones. As can be seen, the largest differences between our algorithm results and the reference products typically appear at the boundaries of the segments corresponding to the ice edge. This indicates that our algorithm is capable of representing the boundaries of regions with different concentrations at a resolution defined by the SAR mosaic resolution. There are still some differences when compared to ice chart concentrations, especially in the coastal zones.
This can be explained by the low resolution of the radiometer data: in the case of a narrow ice zone near the coast, the ice concentration is highly underestimated by our algorithm. This may be due to the narrow shape of the ice zone along the coastline, which is excluded by the land masking. These concentrations are better estimated by the $3 \mathrm{~km}$ ASI algorithm, indicating that using higher-resolution AMSR-2 data in our algorithm would also probably improve the estimation in the coastal areas. Also, there seem to be some low-concentration areas produced by our algorithm in areas where the ice charts indicate much higher concentrations, for example the area in Fig. 3, approximately around $64^{\circ} \mathrm{N}, 23^{\circ} \mathrm{E}$. It is very difficult to say whether these segments are due to the more precise distinguishing capability of the algorithm (when the ice analyst has given a concentration value for a larger polygon) or to an estimation error (e.g. due to wet snow or water over the ice). In any case, the use of segment-wise brightness temperature value modes seems to improve the ice concentration estimation significantly compared to radiometer data alone with respect to the gridded ice chart concentrations. It can be expected that applying our algorithm to radiometer data at a higher resolution would lead to even better results; then the concentration of smaller ice areas and concentration areas near coasts would be estimated better.

\section{Conclusions}

We have developed an algorithm combining sea ice concentration estimates based on radiometer data and SAR segmentation to yield high-resolution ice concentration estimates. The radiometer-based estimates are computed in the resolution defined by the radiometer data, and then the estimates are updated using the SAR segmentation. The results are given in the resolution defined by the SAR segmentation. In this experiment we have used an MLP neural network to estimate ice concentration from the radiometer data, but, in principle, any ice concentration estimation could be combined to the SAR segmentation.

The estimation results were compared to the FMI ice charts and ASI algorithm estimates for an independent test data set. The differences when compared to FMI ice chart ice concentrations and to ASI ice concentrations were on average relatively small. The differences were a bit smaller for the FMI ice chart data than for the ASI data. Because the FMI ice charts have been used as a training data set, the algorithm has been adapted to the ice concentration estimates given by the FMI ice analysts in the ice charts. The most significant differences when compared to the reference data sets occur at the boundaries of different ice concentration zones and near the sea-land boundaries.

Also, a visual comparison between the radiometer-based products (ASI, AMSR-2 bootstrap) and our combined product for the Baltic Sea was generally in good agreement. The differences for our two Arctic test area image mosaics were 
larger than for the Baltic Sea, but even using only Baltic Sea training data the results were surprisingly good, and we expect better results after training the MLP with Arctic data. (Because we do not have high-resolution ice charts available over the Arctic, we have to use suitably filtered radiometerbased ice concentration data in the training.) The comparison results are presented in detail in Sect. 4.

If fresh SAR data are available, it is useful to refine radiometer-based ice concentration estimates using the SAR data to refine the estimates. This is necessary, for example, for navigation to get concentration estimates on a scale closer to the ship scale.

Due to the promising results we are going to implement this kind of operational ice concentration estimation algorithm at FMI in the near future. In our operational algorithm we are going to use the AMSR-2 data sampled at the resolution defined by the AMSR-2 $89 \mathrm{GHz}$ channel, as in the ASI algorithm, to further improve the estimates. The algorithm will mostly rely on the European Space Agency's (ESA) Sentinel-1 C-band SAR data and will be run over the Baltic Sea and some Arctic areas for which SAR data are available.

Acknowledgements. Thanks to L. Kaleschke for his constructive comments and editing the manuscript.

Edited by: L. Kaleschke

\section{References}

Beitsch, A., Kaleschke, L., and Kern, S.: AMSR2 ASI 3.125 km Sea Ice Concentration Data, V0.1, Institute of Oceanography, University of Hamburg, Germany, digital media, available at: ftp: //ftp-projects.zmaw.de/seaice/ (last access: 31 July 2014), 2013.

Beitsch, A., Kaleschke, L., and Kern, S.: The February 2013 Arctic Sea Ice Fracture in the Beaufort Sea - a case study for two different AMSR2 sea ice concentration algorithms, Remote Sensing, 6, 3841-3856, 2014.

Berg, A.: Spaceborne SAR in Sea Ice Monitoring: Algorithm Development and Validation for the Baltic Sea, Licentiate thesis, Technical Report 47L, Chalmers University of Technology, Gothenburg, Sweden, 2011.

Berthod, M., Kato, Z., Yu, S., and Zerubia, J.: Bayesian image classification using Markov Random Fields, Image Vision Comput., 14, 285-295, 1996.

Besag, J.: On the statistical analysis of dirty pictures, J. Roy. Stat. Soc. B, 48, 259-302, 1986.

Bovith, T. and Andersen, S.: Sea Ice Concentration from SinglePolarized SAR data using Second-Order Grey Level Statistics and Learning Vector Quantization, Scientific Report 05-04, Danish Meteorological Institute, Copenhagen, Denmark, 2005.

Cavalieri, D. J., Gloersen, P., and Campbell, W. J.: Determination of sea ice parameters with the NIMBUS 7 SMMR, J. Geophys. Res., 89, 5355-5369, 1984.

Cavalieri, D. J., Markus, T., Hall, D. K., Gasiewski, A. J., Klein, M., and Ivanoff, A.: Assessment of EOS Aqua AMSR-E Arctic Sea
Ice Concentrations Using Landsat-7 and Airborne Microwave Imagery, IEEE T. Geosci. Remote, 44, 3057-3069, 2006.

Clausi, D. A.: Comparison and fusion of co-occurrence, Gabor, and MRF texture features for classification of SAR sea ice imagery, Atmos. Oceans, 39, 183-194, 2001.

Clausi, D. A. and Jernigan, M. E.: Designing Gabor filters for optimal texture separability, Pattern Recogn., 33, 1835-1849, 2000.

Comiso, J. C.: Characteristics of Arctic winter sea ice from satellite multispectral microwave observations, J. Geophys. Res., 91, 975-994, 1986.

Comiso, J. C.: SSM/I Sea Ice Concentrations Using the Bootstrap Algorithm, NASA Reference Publication/Goddard Space Flight Center 1380, Greenbelt, Maryland, USA, 1995.

Deng, H. and Clausi, D. A.: Gaussian MRF rotation-invariant features for image classification, IEEE T. Pattern Anal., 26, 951955, 2004.

Deng, H. and Clausi, D. A.: Unsupervised segmentation of synthetic aperture radar sea ice imagery using a novel Markov random field model, IEEE T. Geosci. Remote, 43, 528-538, 2005.

Dokken, S. T., Hakansson, B., and Askne, J.: Inter-comparison of Arctic Sea ice concentration using RADARSAT, ERS, SSM/I and in-situ data, Can. J. Remote Sens., 26, 521-536, 2000.

Drüe, C. and Heinemann, G.: High-resolution maps of the sea-ice concentration from MODIS satellite data, Geophys. Res. Lett., 31, L20403, doi:10.1029/2004GL020808, 2004.

Haralick, R. M., Shanmugam, K., and Dinstein, I.: Textural features for image classification, IEEE T. Syst. Man Cyb., SMC-3, 610621, 1973.

Haykin, S. S.: Neural Networks, a Comprehensive Foundation, 2nd Edn., Prentice Hall, Upper Saddle River, N.J., USA, 183-196, 1999.

Hornik, K., Stinchcombe, M., and White, H.: Multilayer feedforward networks are universal approximators, Neural Networks, 2, 359-366, 1989.

JAXA: Descriptions of GCOM-W1 AMSR2 Level 1R and Level 2 Algorithms, Rev.A, available at: http://suzaku.eorc.jaxa.jp/ GCOM_W/data/data_w_algorithm.html (last access: 31 July 2014), 2013.

Kaleschke, L. and Kern, S.: ERS-2 SAR Image Analysis for Sea Ice Classification in the Marginal Ice Zone, Proc. IEEE International geoscience and remote sensing symposium 2000 (IGARSS 2000), V, 3038-3040, 24-28 July, 2000, Honolulu, Hawaii, USA, 2000.

Kaleschke, L., Lupkes, C., Vihma, T., Haarpaintner, J., Bochert, A., Hartmann, J., and Heygster, G.: SSM/I sea ice remote sensing for mesoscale ocean-atmosphere interaction analysis, Can. J. Remote Sens., 27, 526-537, 2001.

Karvonen, J.: Baltic Sea ice concentration estimation based on CBand HH-Polarized SAR data, IEEE J. Sel. Top. Appl., 5, 1874 1884, doi:10.1109/JSTARS.2012.2209199, 2012.

Karvonen, J.: Baltic Sea ice concentration estimation based on CBand Dual-Polarized SAR data, IEEE T. Geosci. Remote, 52, 5558-5566, doi:10.1109/TGRS.2013.2290331, 2014.

Karvonen, J., Cheng, B., and Simila, M.: Ice Thickness Charts Produced by C-Band SAR Imagery and HIGHTSI Thermodynamic Ice Model, Proc. of the Sixth Workshop on Baltic Sea Ice Climate, 71-81, 25-28 August 2008, Lammi, Finland 2008.

Karvonen, J., Cheng, B., Vihma, T., Arkett, M., and Carrieres, T.: A method for sea ice thickness and concentration analysis based 
on SAR data and a thermodynamic model, The Cryosphere, 6 , 1507-1526, doi:10.5194/tc-6-1507-2012, 2012.

Kato, Z., Zerubia, J., and Berthod, M.: Satellite Image Classification Using a Modified Metropolis Dynamics, in: Proceedings of IEEE International Conference on Acoustics, Speech and Signal Processing (ICASSP 92), 3, 23-26 March 1992, San Francisco, California, USA, 573-576, March, 1992.

Leigh, S., Wang, Z., and Clausi, D. A.: Automated ice-water classification using dual polarization SAR satellite imagery, IEEE T. Geosci. Remote, 52, 5529-5539, 2014.

Maass, N. and Kaleschke, L.: Improving passive microwave sea ice concentration algorithms for coastal areas: applications to the Baltic Sea, Tellus, 62A, 393-410, 2010.

MacQueen, J. B.: Some Methods for classification and Analysis of Multivariate Observations, in: Proceedings of 5th Berkeley Symposium on Mathematical Statistics and Probability, 21 June-18 July 1965 and 27 December 1965-7 June 1966, University of California Press., 281-297, 1967.

Maeda, T.: AMSR2 L1R Product, JAXA, available at: http://suzaku. eorc.jaxa.jp/GCOM_W/materials/product/AMSR2_L1R.pdf (last access: 31 July 2014), 2013.

Maillard, P., Clausi, D. A., and Deng, H.: Map-guided sea ice segmentation and classification using SAR imagery and a MRF segmentation scheme, IEEE T. Geosci. Remote, 43, 2940-2951, 2005.

Makynen, M., Manninen, T., Simila, M., Karvonen, J., and Hallikainen, M.: Incidence angle dependence of the statistical properties of the C-Band HH-Polarization backscattering signatures of the Baltic Sea Ice, IEEE T. Geosci. Remote, 40, 2593-2605, 2002 .
Ochilov, S. and Clausi, D. A.: Operational SAR sea-ice classification, IEEE T. Geosci. Remote, 50, 4397-4408, 2012.

Pichler, O., Teuner, A. S., and Hosticka, B. J.: A comparison of texture feature extraction using adaptive gabor filtering, pyramidal and tree structured wavelet transforms, Pattern Recogn., 29, 733-742, 1996.

Rue, H. and Held, L.: Gaussian Markov Random Fields: Theory and Applications, CRC Press, 2005.

Spreen, G., Kaleschke, L., and Heygster, G.: Sea ice remote sensing using AMSR-E 89-GHz channels, J. Geophys. Res., 113, C02S03, doi:10.1029/2005JC003384, 2008.

Steffen, K., Key, J., Cavalieri, D. J., Comiso, J., Gloersen, P., St. Germain, K., and Rubinstein, I.: The estimation of geophysical parameters using passive microwave algorithms, Ch. 10, in: Microwave Remote Sensing of Sea Ice, edited by: Carsey, F. D., American Geophysical Society/Wiley, Washington D.C., USA, 201-231, 1992.

Yu, Q. and Clausi, D. A.: SAR sea-ice image analysis based on iterative region growing using semantics, IEEE T. Geosci. Remote, 45, 3919-3931, 2007.

Wessel, P. and Smith, W. H. F.: A global self-consistent, hierarchical, high-resolution shoreline database, J. Geophys. Res., 101, 8741-8743, 1996. 